\title{
IoT A GATEWAY FOR ACCEPTANCE: THE POTENTIAL FOR BROAD PURPOSE IMPLANT DEVICES
}

\author{
Jennifer L. Breese, Penn State University, jzb545@psu.edu \\ Tatiana Zwerling, Penn State University, tatiana@psu.edu
}

\begin{abstract}
While the term Internet of Things (IoT) has become commonplace in both technologists and the average consumer vernacular, implications in terms of usage in implanted devices and the potential for changing social norms have been largely overlooked in literature. The goal of this secondary literature review was to explore two questions through the frame of the Technology Acceptance Model, Technology Innovation and Diffusion Model, Agency Theory, and additional background literature. First, while there are several obvious reasons to adopt a medical implant for quality of life purposes, could the election to implant a non-medically necessary spread through the Technology Innovation and Diffusion model? Secondly, could perceptions of timesaving's influence certain populations to accept technology for ease of use thwarting privacy for convenience?
\end{abstract}

Keywords: Internet of Things (IOT), Implant Devices, Implant Acceptance, Implant Technology in Medical Devices

\section{INTRODUCTION}

The goal of this secondary literature review was to explore two questions through several frameworks: the Technology Acceptance Model, Technology Innovation and Diffusion Model, Agency Theory, and additional background literature. First, while there are several obvious reasons to adopt a medical implant technologies for quality of life purposes; could the election to have a non-medically necessary implant spread through the Technology Innovation and Diffusion model? Secondly, could perceptions of timesaving's influence certain populations to accept technology for ease of use thwarting privacy for perception of convenience?

While the term Internet of Things (IoT) has become ingrained the everyday vernacular for both technologists and the average consumer, implications in terms of explorations regarding changes in implanted usage and social norms for accepted use have been largely overlooked in literature. The ways we receive accept new technologies, the speed of change, and the sources we rely on and trust to provide transparency are changing exponentially; IoT service providers realize that consumers want more benefits despite their dual concerns about privacy. There exists a tendency for consumers to lower the weight of perceived privacy risks in pursuit of perceived benefits Kim et al., (2019) benefits of new technology adoption may not provide life or even time savers. IoT medical implant devices, an example of lifesaving uses as defined by Catherwood et al. (2015) these devices are prescribed and fitted by medical practitioners replacing missing biological structure, sustaining life and alleviating the symptoms of chronic illness. Non-life saving and debatable timesaving uses for IoT implanted technology are described as 'human tagging' (Voas \& Kshetri, 2017). Human tagging is a non-implant technology, but raises serious privacy including data ownership concerns. Should we be able to go about everyday activities without companies and the government having the ability to track our destination, patterns, and activities? Voas and Kshetri (2017) cite examples of 'human tagging' technology as smartcards and security cards for entrance. Pisani (2020) describes Amazon abbreviated checkouts where a desire among certain populations and perhaps a further willingness to comply has been created by the 2020 Covid-19 crisis need for social distancing. Pisani (2020) reports the Amazon cashier-less store where you can grab your items with an app and simply walk out. This technology has been criticized for leaving out lower socio-economic participants many do not have credit or bank accounts, but even 7-Eleven is also testing this type of technology. While the cashierless checkout is an example of an external digital application of the IoT this could easily be adapted and subsequent may result in adopted technology innovations for micro-chip implant technologies. However, navigating the 
technology innovation and adoption landscape can be difficult and often predictions of successful future technologies misstep.

\section{BACKGROUND AND IMPLICATIONS (LITERATURE REVIEW)}

\section{What is the Internet of Things (IoT)}

The Internet of Things (IoT) has become a ubiquitous phrase, familiar to most, especially those working in technology fields. However, what it refers to has morphed over time since the term was initially coined in 1999 by Kevin Ashton (Ashton, 2009). Ashton initially used the term "Internet of Things" to refer to the then-nascent use of radio frequency identification (RFID) sensors in supply chain logistics (Ashton, 2009). The Internet of Things has made it possible for "computers [to] sense things for themselves" (Gabbai, 2015), which was Ashton's goal all along. Ashton (2009) noted that computers and the Internet are dependent on humans to provide information. However, given that "people have limited time, attention and accuracy," he pointed out people are not the best at taking data from the physical world and translating it into digital data (p 4986). Ashton (2009) further elaborated that the objective is to use data gathered by sensors to improve processes and "reduce waste, loss, and cost" (p 4986). Ashton (2009) noted that while he may have coined the terminology "Internet of Things," he does not control how others may imbue meaning into the phrase. In IEEE's 2015 report on IoT, which sought to define the burgeoning technology, researchers gave this definition: "A network of items - each embedded with sensors - which are connected to the Internet" (Minerva et al., 2015, p 10). The Oxford English Dictionary defines IoT as "a proposed development of the internet in which many everyday objects are embedded with microchips giving them network connectivity, allowing them to send and receive data" (“Internet, n.," 2020) Murer first blogged in 2010, on the then-fledgling website theinternetofthings.eu:

the best definition for the Internet of Things would be: "an open and comprehensive network of intelligent objects that have the capacity to auto-organize, share information, data and resources, reacting and acting in [the] face of situations and changes in the environment."

While the phrase Internet of Things is more prevalently used, it does have some sister phrases which are used as well: Internet of Objects, Embedded Intelligence, Web of Things, Pervasive Computing, Ubiquitous Computing, Internet of Connected Devices, Cyber Physical Systems, Human Computer Interaction, and Wireless Sensor Networks (Irmak \& Bozdal, 2018). Regardless of terminology, as technology has continued to become smaller and more powerful, IoT has become more pervasive, throughout a myriad of industries as well as home and personal computing. Minerva et al. (2015) documented the widespread use of IoT devices within the following areas: consumer equipment providers, consumers, Information Communication Technologies infrastructure providers, regulators, logistics companies, public transport companies, city authorities, manufacturing industries, healthcare, insurance companies, appliance providers, facility management, retail stores, application developers, utilities, and automation equipment providers. Merely three years later, Wired describes the IoT landscape as, "smart toasters, connected rectal thermometers and fitness collars for dogs," these being some of the more mundane pieces (Burgess, 2018). Gartner (2016, 2017) forecasted that the total number of connected, or networked, 'things' would reach 20.4 billion by 2020 .

While IoT is broadly used to describe a myriad of networked devices, the moniker has begun to adjust according to each respective industry. Some examples of this are: Industrial IoT (IIoT), Collaborative IoT, Cellular IoT (both are C-IoT), Cognitive IoT (CIoT), Internet of Musical Things (IoMusT), and Internet of Medical Things or Medical IoT (which use IoMT and MIoT, respectively - note - MIoT is also used for mobile IoT and Multiple IoTs), and Social IoT (Boyes et al., 2018; Belkacem et al., 2019; Sari et al., 2020; Li et al., 2016; Turchet et al., 2018; Darwish et al., 2017; Tian et al., 2020; Baldassarre et al., 2019). This is far from an exhaustive list of abbreviations for the burgeoning market of IoT devices. The vast number of abbreviations added to the IoT lexicon is indicative of how thoroughly IoT has infiltrated such a wide variety of industries. 


\section{American Obsession with Technology, Belonging, and Scarcity of Time}

This section addresses the socio-technical reasons that drive acceptance within a culture. A culture is based upon openness, trust, respect, collaboration, shared meanings, and resources reflects a mutual understanding of goals, objectives and purpose. How these values and mission apply to 'group-think' in a techno-virtual environment is a consideration. Technology and culture are interwoven, implicitly and explicitly intimately connected and linked by communities of practice, semiotics, signs, cues, and coded and decoded language (English, digital, and/or otherwise) (Breese-Vitelli \& Borkovich, 2013). The socio-technical theory Berger and Luckmann (1966) describe as the interaction between people and technology in the workplace and social informatics using a social construction of reality. Groups in a system over time create meaning, roles and an interdependent reciprocal roles based on mental constructs developed for one another. Reality is social constructed (Berger \& Luckmann, 1966). 'Technopoly' a word used by Postman (1992) to describe the primary, and perhaps only, goal of labor in increased efficiency and technical calculation in favor of human judgment. Postman (1992) states the American 'Technopoly' has embraced the computer in a mindless hurried manner usurping powers and forcing mind-sets a fully attentive culture might deny it.

Will people agree to implanted technology based on a company culture, acceptance of technology among social ties, or for the greater good as in a pandemic? The Greek philosopher, Seneca [c. 4 BC - 65 AD], stated "Time is our most scarce resource," and many are choose to adopt new technologies as a remedy to lack of time. The Amazon check out technology is certainly one aspect of willingness to forego privacy for convenience and there are many others. The growing acceptance of wearable technologies is leading to term the 'connected human' which describes the intimate nature between humans and machines (Benioff, 2014). Goffman (1959) discussed behavior in terms of social psychology where one is always performing and the performance is socialized, molded, and conformed to fit societal expectations. The expressive bias for the performance within the 'norms' then becomes the reality (Goffman, 1959). The perception that new technology is better, faster, leads to adoption as an emblematic symbol of belonging.

\section{Agency}

Agency theory, as defined by Jensen and Meckling (1976) evaluates the developing relationship in regard to economic exchange when an individual (principal) readily concedes their authority to another (agent) to facilitate actions in his or her name so that the wealth obtained by the principal is benefited based on decisions adopted by the agent. According to Jensen and Meckling, when ownership is separated from control, a cost to the principal, known as agency cost, can require expensive mechanisms to control these costs. Agency costs result when agents pursue interest that may not coincide with those of the principal. These interests may be aligned based on mutual incentives between principal and agent and can result in a reduction in agency cost.

While instances exist where interest may align between agent and principal, agency theory also explores conflicts of interest in the form of optimal contracts which are designed to correct these conflicts (Eisenhardt, 1989). According to Keeley (1980), one of the most efficient types of contract, are most beneficial and least costly to the principal and is formed with complete information where the effort expended by the agent is known (Demski \& Feldman, 1978). In instances where an agent has more information than the principal, the agent is free to act on their own behalf and further their own self-interest (Fong \& Tosi, 2007). A second type of contract emphasizes, ensuring that the outcomes desired by the principal are directly tied to the agent's compensation (Baiman, 1990; Riordan \& Sappington, 1987).

In summary, agreement of terms and conditions should only be valid when the principle (consumer) both understands and concedes their authority to the agent. Blind agreement without a true understanding between the principle and the agent in an agreement needs a further test of understanding in addition to a check box at the end of a scroll of lengthy complicated legal verbiage.

\section{Implant Technology in Medical Devices}

In the healthcare field, the rise of IoT devices has led to the creation of what is known as Wireless Body Area Networks (WBAN) (Sharavanan et al., 2018). These WBAN consist of numerous sensors meant to monitor patients and their vital signs. These sensors are either placed externally on the patient, or in some cases, the sensors are implanted 


\section{Issues in Information Systems}

Volume 21, Issue 4, pp. 114-122, 2020

(Sharavanan et al., 2018). These sensors are crucial in providing healthcare practitioners with accurate and timely information on everything from a patient's blood pressure to blood glucose, EEG, and respiration (Sharavanan et al., 2018). Some of these devices, especially those that are implanted, are lifesaving devices such as medication pumps, pacemakers, heart defibrillators, cochlear implants, deep brain stimulation devices for patients with Parkinson's disease tremors and seizures, and retinal implants. Others are nascent devices that are being developed as implants that will aid those patients with persistent psychiatric illness like depression (Strickland, 2014). Medical implants as defined by Catherwood et al. (2015) are those that "are prescribed and fitted by medical practitioners for the purpose of replacing missing biological structure, sustaining life and/or alleviating the symptoms of chronic illness."

Outside of medical devices, there are also numerous instances of implanted devices that are primarily used for "convenience, communication, entertainment, shopping, and security" (Catherwood et al., 2015). VeriChip was an RFID device initially used within the medical arena specifically as a device that would allow patients to carry their medical history on an implanted device. VeriChip then became known as a secondary means of identity verification, or in some cases, was used by elite nightclubs for customers to gain VIP access to the clubs allowing customers to be greeted by name and have their favorite drinks ready for them upon entering (Foster \& Jaeger, 2007). There is also an instance of a brain-computer interface developed in which subjects wearing the small, unobtrusive electrode system were able to control a computer solely using the subjects' thought (Norton et al., 2015). Further, discussed in the literature is an electronic skin tattoo that can be used with a mobile communication device and contact lenses that can magnify sight up to three times through the act of winking (Alberth, 2013; Macrae, 2015; Catherwood et al., 2015).

While there are several obvious reasons to adopt a medical implant for quality of life purposes, the reasons to elect to have an implant that is not medically necessary is not always as clear. Catherwood et al. (2015) make the case for human enhancements that would allow people to become, essentially, superhuman. In an era where technology has already infiltrated daily life, the augmentation of human capacity through the use of implantable technology seems to be the next logical progression. Catherwood et al. (2015) note that there is already a large transhumanist movement "who wish to enhance and repair their bodies indefinitely using advanced technology." As there are proponents for the transhumanist movement, there are also those that call into question the ethics of such potential actions. Miah (2009) explores the ethical dilemma in depth discussing the differences between therapeutic interventions and those that could be characterized solely as "enhancements."

Catherwood et al. (2015) also explored the potential hazards of the use of implanted devices. They cite the looming fear that people have for being "chipped and enslaved" along with discussing the concerns of individual human rights being violated should someone be tracked without their consent which is something the authors will note already occurs with smartphones (Reisinger, 2018). Another concern raised by Catherwood et al (2015) is the potential issue of employer surveillance and the possible misuse by employers to track the whereabouts of employees during the workday and to use employee tracking for benchmarking and performance related benefits. There is also the concern about whether implants are safe for use. Catherwood et al (2015) also discuss cases of RFID chips causing cancer and even VeriChip had multiple health risks associated with its product.

\section{THEORETICAL PERSPECTIVES}

\section{Technology Acceptance Model}

This exploration intends to use existing models for technology acceptance and the explanation of various dynamic factors contributing to adoption or otherwise new uses of technology to frame the discussion. The first lens is that of the Technology Acceptance Model (TAM) which specifies the usage of information technology determined by beliefs a user holds about the perceived usefulness (PU) and perceived ease-of-use of the technology (PEU) first proposed by Davis (1989). Other models have been proposed and much criticism has been discussed in related literature for the broad nature and lack of specificity in the TAM model; however, Chutter (2009) argues its foundational nature in Information Systems literature regardless of criticisms in the field. 
Davis (1989) intended to predict and explain technology usage behavior factors which lead to acceptance or rejection through integration of technological and organizational behavior concepts. Interaction between humans and technologies are influenced by several social, psychological factors and characteristics (Taiwo \& Downe, 2013). This paper brings into question what constitutes an organizational and or societal, "nature."

\section{Technology Innovation and Diffusion Model}

While the Technology Acceptance Model (TAM) attempts to address the behavioral intention of users and their adoption decisions, Diffusion of Innovation Model (DOI) looks at the advantages, complexity, and compatibility of innovations. Literature has drawn a connection between the two combining the two models. Essentially, the extension of the model is to examine how other factors e.g. perceived use influence behavior with technological innovation (Lee, Hsieh, \& Hsu, 2011).

The Innovation Diffusion Theory developed by Rogers (1962) and expanded on in Rogers (1983; 1985) described innovation as an idea, process, object, or practice that can be considered to be new; further, diffusion, is the process by which innovation gets into the social system. This theory is considered to be the permanent theory of acceptance of innovation and is relevant for both an individual or organizational context (Yusuf \& Derus, 2013). The model has helped explain technology adoption for over 50 years. Rogers' cites five determinants for the rate of innovation affecting adoption and acceptance behavior; relative advantage, compatibility, complexity, trialability, and observability. Rogers (2003) suggests adopters of innovation can be classified according to the time and ease to adoption. Figure 1 below is a graphical depiction of the five adopter classifications with the typical adoption distribution; Innovators, Early Adopters, Early Majority, Late Majority, and Laggards. Innovators are the most 'venturesome' they are out in-front experiments, communicating, and perhaps debugging new technologies. Early adopters are 'highly respect' in their respective fields or community; they provide guidance and influence to the critical mass. The early majority is representative of the beginning of the majority who can be swayed to adopt earlier than the average, but are known to deliberate before the adoption decision. Late majority adopters are skeptical and follow suite only both early majority adopters and the application of peer pressure. The final adopter is the Laggard, who is a 'traditionalist' suspicious of change and well behind others with regard to awareness of the innovation. (Rogers, 2003). Will the adoption and acceptance of life-saving medical implant devices lead to adoption through the model from Innovators to Laggards; further, with the adoption of these devices lead to adoption of other similar devices that are not life-saving?

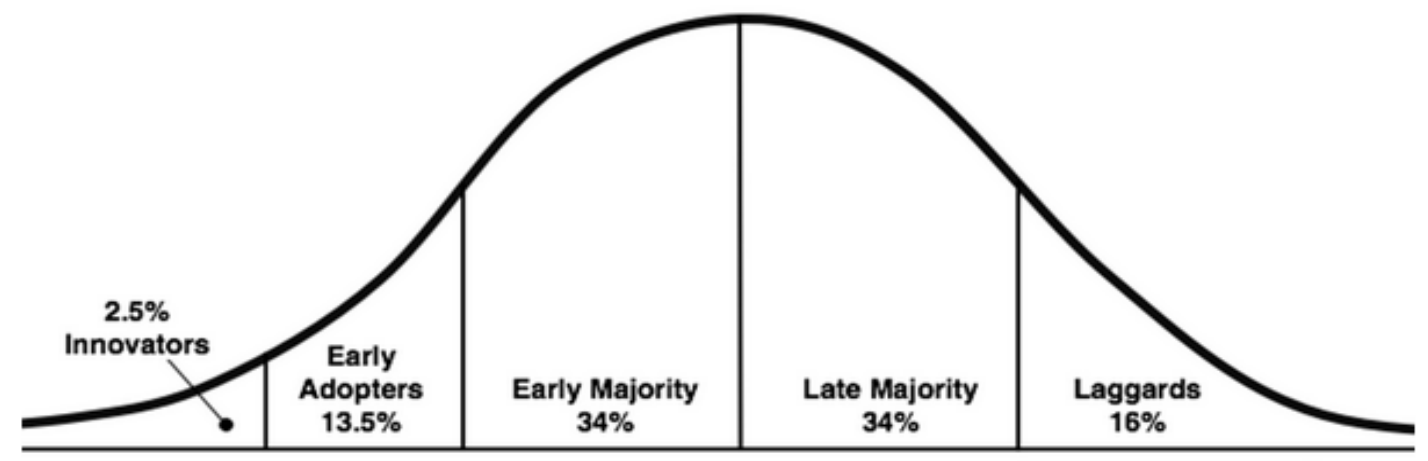

Figure 1. Rogers' Diffusion of Innovation Categories

\section{LIMITATIONS AND FUTURE APPLICATIONS}

It is very common to see the term IoT in reference to new technology applications to make everyday things 'easier.' While addressing the concept the scope of data collection and infringement of privacy are oft overlooked by the consumer. The breadth of the transfer and use of such data must be understood and mechanism(s) must exist for the 


\section{Issues in Information Systems}

Volume 21, Issue 4, pp. 114-122, 2020

protection of said data and the absolute ability to opt out or not share data that flows via the pipeline of IoT. Once the technology becomes mandatory it adds not only to the digital divide concept but fuels a hotly contested political climate. We plan to further explore these concepts through a quantitative investigate creating a taxonomy of those are willing to give up their privacy for medical necessity, simply for convenience, and those who readily surrender their "Agency" for lack of understanding.

\section{CONCLUSIONS}

It was expected that a push back regarding implanted devices would occur among the general population. It is not clear whether adoption of implanted devices for the purposes of medical necessity will accelerate the adoption or impact the perception of 'need' to include elective applications. Whether the acceptance of medical implant devices will translate to adoption in non-necessity areas and perhaps 'superhuman,' medical applications remains to be seen. Some additional considerations are increased acceptance and adoption based on convenience and finally adoption questioning free will and 'Agency' adoption when mandated or the terms and conditions are not well understood by the actor. Mandated implants seem to fulfill a dystopian futuristic; however, many restrictions, tracing, and technology solutions were implemented during the Covid-19 crisis many did not anticipate. This technology innovation and adoption through IoT implant device could continue through the current accepted medical need and bleed over to 'regular' life for non-life-threatening biological applications. The American obsession for time and 'workarounds' could lead to broader technology acceptance and adoption which arguably may not even save much time.

\section{REFERENCES}

Alberth Jr., W. P. (2013). Coupling an electronic skin tattoo to a mobile communication device (United States Patent No. US20130297301A1). https://patents.google.com/patent/US20130297301A1/en

Ashton, K. (2009). That “Internet of Things” Thing. RFID Journal, 4986.

Baiman, S. 1990. Agency research in managerial accounting: A second look. Accounting, Organizations, and Society, 15: 341-371.

Baldassarre, G., Lo Giudice, P., Musarella, L., \& Ursino, D. (2019). The MIoT paradigm: Main features and an "ad-hoc" crawler. Future Generation Computer Systems, 92, $29-42$. https://doi.org/10.1016/j.future.2018.09.015

Belkacem, I., Nait-Bahloul, S., Sauveron, D. (2019). Enhancing dependability through profiling in the collaborative internet of things. Multimedia Tools and Applications; Dordrecht, 78(3), 2983-3007. http://dx.doi.org.ezaccess.libraries.psu.edu/10.1007/s11042-017-54311

Berger, P. L., \& Luckmann, T. (1966). The social construction of reality. New York: Anchor Books.

Boyes, H., Hallaq, B., Cunningham, J., \& Watson, T. (2018). The industrial internet of things (IIoT): An analysis framework. Computers in Industry, 101, 1-12. https://doi.org/10.1016/j.compind.2018.04.015

Breese-Vitelli, J., \& Borkovich, D. J. (2013). Mobile technology culture and its impact on college students' local news viewing behavior. Issues in Information Systems, 14(2), 400-410.

Burgess, M. (2018, February 16). What is the Internet of Things? WIRED explains. Wired UK. https://www.wired.co.uk/article/internet-of-things-what-is-explained-iot

Catherwood, P. A., Finlay, D. D., \& McLaughlin, J. A. D. (2015). Subcutaneous body area networks: A SWOT analysis. 2015 IEEE International Symposium on Technology and Society (ISTAS), 1-8.

https://doi.org/10.1109/ISTAS.2015.7439414 
Chuttur M.Y. (2009). Overview of the Technology Acceptance Model: Origins, Developments and Future Directions, Indiana University, USA. Sprouts: Working Papers on Information Systems, 9(37). http://sprouts.aisnet.org/9-37

Darwish, S., Nouretdinov, I., \& Wolthusen, S. D. (2017). Towards Composable Threat Assessment for Medical IoT (MIoT). Procedia Computer Science, 113, 627-632.

Davis, F. D. (1986). A Technology acceptance model for empirically testing new end-user information systems: Theory and results. Doctoral Dissertation, MIT Sloan School of Management, Cambridge.

Davis, F. D. (1989). Perceived usefulness, perceived ease of use, and user acceptance of information technology. MIS Quarterly, 13(3), 319-340.

Davis, F. D., Bagozzi, R. P., \& Warshaw, P. R. (1989). User acceptance of computer technology: A comparison of two theoretical models. Management Science, 25 (8), 982-1003.

Davis, F. D. \& Venkatesh, V. (2004). Toward preprototype user acceptance testing of new information systems for software project management. Engineering Management, IEEE Transactions, 51(1), 31-46.

Demski, J. S., \& Feltham, G. A. 1978. Economic incentives in budgetary control systems. The Accounting Review, 53: 336-359.

Eisenhardt, K. M. (1989). Agency theory: An assessment and review. Academy of Management Review, 14(1), 5774.

Evangelos A., K., Nikolaos D., T., \& Anthony C., B. (2011). Integrating RFIDs and Smart Objects into a Unified Internet of Things Architecture. Advances in Internet of Things, 2011. https://doi.org/10.4236/ait.2011.11002

Fong, Eric A. \& Tosi Jr., Henry L., 2007. Effort, Performance, and Conscientiousness: An Agency Theory Perspective. Journal of Management, 33: 163.

Foster, K. R., \& Jaeger, J. (2007). RFID Inside. IEEE Spectrum, 44(3), 24-29. https://doi.org/10.1109/MSPEC.2007.323430

Gabbai, A. (2015, January). Kevin Ashton Describes "the Internet of Things.” Smithsonian Magazine. https://www.smithsonianmag.com/innovation/kevin-ashton-describes-the internet-of-things-180953749/

Gartner Says 8.4 Billion Connected “Things” Will Be in Use in 2017, Up 31 Percent From 2016. (2017, February 7). Gartner. https://www.gartner.com/en/newsroom/press releases/2017-02-07-gartner-says-8-billion-connected-things-will-be-in-use-in-2017-up 31-percent-from-2016

Goffman, E. (1959). The presentation of self in everyday life. New York: Anchor Books.

Irmak, E., \& Bozdal, M. (2018). Internet of Things (IoT): The Most Up-To-Date Challenges, Architectures, Emerging Trends and Potential Opportunities. International Journal of Computer Applications, 179(40), 20-27. https://doi.org/10.5120/ijca2018916946

Jensen, M. \& Meckling, W. 1976. Theory of the firm: Managerial behavior, agency cost, and capital structure. Journal of Financial Economics, 3: 305-360.

Kim, D., Park, K., Park, Y., \& Ahn, J.-H. (2019). Willingness to provide personal information: Perspective of privacy calculus in IoT services. Computers in Human Behavior, 92, 273-281. doi: 10.1016/j.chb.2018.11.022 
Lee, Yi-Hsuan \& Hsieh, Yi-Chuan \& Hsu, Chia-Ning. (2011). Adding Innovation Diffusion Theory to the Technology Acceptance Model: Supporting Employees' Intentions to use E-Learning Systems. Educational Technology \& Society. 14. 124-137.

Li, Z., Jing, T., Ma, L., Huo, Y., \& Qian, J. (2016). Worst-Case Cooperative Jamming for Secure Communications in CIoT Networks. Sensors; Basel, 16(3), 339. http://dx.doi.org.ezaccess.libraries.psu.edu/10.3390/s16030339

Madakam, S., Ramaswamy, R., \& Tripathi, S. (2015). Internet of Things (IoT): A Literature Review. Journal of Computer and Communications, 3(5), 164-173. https://doi.org/10.4236/jcc.2015.35021

Macrae, F. (2015, February 13). Contact lenses magnify words on demand and are controlled by winking. Mail Online. https://www.dailymail.co.uk/sciencetech/article-2952588/Now-SUPERHERO-vision-Contactlenses-magnify-words-THREE-FOLD-controlled-winking.html

Miah, A. (2009). Engineering greater resilience or radical transhuman enhancement? 2009 IET Bionic Health: Next Generation Implants, Prosthetics and Devices, 1-2. https://doi.org/10.1049/ic.2009.0190

Minerva, R., Biru, A., \& Rotondi, D. (2015). IEEE IoT Towards a Definition of the Internet of Things (IoT), Revision 1. 1-86.

Murer, R. (2010, November 25). Internet of Things - Fundamentals by Ricardo Murer | the internet of things. https://www.theinternetofthings.eu/content/internet-things \%E2\%80\%93-fundamentals-ricardo-murer

Norton, J. J. S., Lee, D. S., Lee, J. W., Lee, W., Kwon, O., Won, P., Jung, S.-Y., Cheng, H., Jeong, J.-W., Akce, A., Umunna, S., Na, I., Kwon, Y. H., Wang, X.-Q., Liu, Z., Paik, U., Huang, Y., Bretl, T., Yeo, W.-H., \& Rogers, J. A. (2015). Soft, curved electrode systems capable of integration on the auricle as a persistent brain-computer interface. Proceedings of the National Academy of Sciences of the United States of America, 112(13), 3920-3925. https://doi.org/10.1073/pnas.1424875112

Pisani, J. (2020, February 25). No checkout needed: Amazon opens cashier-less grocery store. Retrieved May 15, 2020, from https://apnews.com/623bd4cd2b6c949d9d9411090447c371

Reisinger, D. (2018, August 13). Google Is Tracking Your Location-Even Without Your Permission, Report Says. Fortune. https://fortune.com/2018/08/13/google-tracking-locations-without-permission/

Riordan, M., \& Sappington, D. 1987. Awarding monopoly franchises. American Economic Review, 77: 375-388.

Rogers, E. M. (1962). The Diffusion of Innovations, 1st ed., New York: Free Press.

Rogers, E. M. (1983). The Diffusion of Innovations, 3rd ed., New York: Free Press.

Rogers, E. M., (1995). Diffusion of Innovations, 4th ed., New York: Free Press.

Sari, R. F., Harwahyu, R., \& Cheng, R.-G. (2020). Load Estimation and Connection Request Barring for Random Access in Massive C-IoT. IEEE Internet of Things Journal, 1-1. https://doi.org/10.1109/JIOT.2020.2968091

Scoble, R., Israel, S., \& Benioff, M. (2014). Age of context: mobile, sensors and the future of privacy. Verenigde Staten van Amerika: Patrick Brewster Press. 
Sharavanan, P. T., Sridharan, D., \& Kumar, R. (2018). A Privacy Preservation Secure Cross Layer Protocol Design for IoT Based Wireless Body Area Networks Using ECDSA Framework. Journal of Medical Systems, 42(10), 196. https://doi.org/10.1007/s10916-018-1050-2

Strickland, E. (2014, May 27). We Will End Disability by Becoming Cyborgs-IEEE Spectrum. IEEE Spectrum: Technology, Engineering, and Science News. https://spectrum.ieee.org/biomedical/bionics/we-will-enddisability-by-becoming-cyborgs

Sundmaeker, H., Guillemin, P., Friess, P., Woelfflé, S., European Commission, \& Directorate General for the Information Society and Media. (2010). Vision and challenges for realising the Internet of things. EUR-OP.

Taiwo, A., \& Downe, A. (2013). The Theory Of User Acceptance And Use Of Technology (UTAUT): A MetaAnalytic Review Of Empirical Findings. Journal of Theoretical and Applied Information Technology, 49(1), 48-58.

Tian, Q., Lin, Y., Guo, X., Wang, J., AlFarraj, O., \& Tolba, A. (2020). An Identity Authentication Method of a MIoT Device Based on Radio Frequency (RF) Fingerprint Technology. Sensors, 20(4), 1213. https://doi.org/10.3390/s20041213

Turchet, L., Fischione, C., Essl, G., Keller, D., \& Barthet, M. (2018). Internet of Musical Things: Vision and Challenges. IEEE Access, 6, 61994-62017. https://doi.org/10.1109/ACCESS.2018.2872625

Voas, J., and Kshetri, L., (2017) Human Tagging, in Computer, 50(10), 78-85.doi: 10.1109/MC.2017.3641646.

Yusuf, M., \& Derus, A. M. (2013). Measurement Model of Corporate Zakat Collection in Malaysia: A Test of Diffusion of Innovation Theory. Humanomics, 29(1), 61-74. 